Cahiers $d u$ MONDE RUSSE

\section{Cahiers du monde russe}

Russie - Empire russe - Union soviétique et États indépendants

$51 / 4 \mid 2010$

Sciences humaines et sociales en Russie à l'Âge d'argent

\title{
Pëtr Bogatyrëv et le théâtre de marionnettes
}

L'émergence d'une sémiotique théâtrale entre Russie et Tchécoslovaquie

Pëtr Bogatyrëv and puppet theater: Emergence of a semiotic theory of the theater between Russia and Czechoslovakia

\section{Céline Trautmann-Waller}

\section{(2) OpenEdition \\ Journals}

Édition électronique

URL : https://journals.openedition.org/monderusse/9207

DOI : $10.4000 /$ monderusse. 9207

ISSN : $1777-5388$

Éditeur

Éditions de l'EHESS

Édition imprimée

Date de publication : 25 novembre 2010

Pagination : 547-563

ISBN : 978-2-7132-2316-7

ISSN : $1252-6576$

Référence électronique

Céline Trautmann-Waller, "Pëtr Bogatyrëv et le théâtre de marionnettes », Cahiers du monde russe [En ligne], 51/4 | 2010, mis en ligne le 20 décembre 2013, consulté le 03 septembre 2022. URL : http:// journals.openedition.org/monderusse/9207; DOI : https://doi.org/10.4000/monderusse.9207

Ce document a été généré automatiquement le 3 septembre 2022.

Tous droits réservés 


\title{
Pëtr Bogatyrëv et le théâtre de marionnettes
}

\author{
L'émergence d'une sémiotique théâtrale entre Russie et \\ Tchécoslovaquie
}

Pëtr Bogatyrëv and puppet theater: Emergence of a semiotic theory of the theater between Russia and Czechoslovakia

\section{Céline Trautmann-Waller}

1 Le cas de Pëtr Grigorevič Bogatyrëv peut paraître particulièrement évocateur de la richesse des rencontres intellectuelles praguoises de l'entre-deux-guerres, dont le Cercle de Prague fut le fruit et l'illustration la plus célèbre. C'est là que le jeune ethnographe russe, qui avait fait partie en 1915 des fondateurs du Cercle linguistique de Moscou et qui n'avait publié, avant son arrivée en 1921, pour l'essentiel que des rapports d'expéditions ethnographiques dans le gouvernement d'Arhangel'sk et une recension, développa une série de chantiers originaux qui concernaient l'ethnographie rurale et l'étude du folklore, la littérature (collecte de manuscrits littéraires russes dans toute la Tchécoslovaquie pour le compte du Musée littéraire d'État de Moscou), la traduction de textes tchèques en russe et l'étude du théâtre populaire.

2 Loin de s'être cantonné à Prague, Bogatyrëv voyagea beaucoup durant ces années 1921 à 1939, de collecte en expédition ethnographique ou en poste d'enseignant, et des recherches récentes ont permis de mieux retracer ses déplacements en Tchécoslovaquie, mais aussi plus largement en Europe. Ses brèves publications dans toute une série de revues praguoises permettent de mieux comprendre comment il chercha à l'époque non seulement à s'insérer dans la vie scientifique tchèque, mais également à découvrir la culture tchèque et plus particulièrement la scène théâtrale qui en constituait depuis un certain temps déjà un élément clé et un moteur. J'en arrive ainsi à celui de ces chantiers sur lequel j'aimerais m'attarder plus longuement dans cet article.

Pendant son séjour à Prague, plus précisément entre les années 1923 et 1931, Bogatyrëv a consacré au théâtre de marionnettes un livre et plusieurs articles, ces derniers commentant pour la plupart des événements concrets liés au théâtre de marionnettes 
dans l'actualité culturelle tchécoslovaque. Il s'agit d'une part du livre Češkij kukol'nyj i russkij narodnyj teatr publié en 1923 par l'OPOJAZ dans la série Sborniki po teorii poètičeskogo jazyka ${ }^{1}$, d'autre part de quatre articles parus en 1927 et en 1929 dans la revue Central'naja Evropa publiée en russe à Prague ${ }^{2}$, de plusieurs articles publiés dans la revue praguoise Loutkár [Le marionnettiste] ${ }^{3}$, dont un article en langue française de 1930 issu d'une communication sur le théatre russe de marionnettes au Congrès des amis des marionnettes qui s'était tenu en octobre de la même année à Liège ${ }^{4}$, et d'un article en langue allemande paru en 1931 dans Dichtung und Wahrheit, supplément de la revue praguoise de langue allemande Prager Presse $e^{5}$, et consacré au théâtre de marionnettes à Münster, ville où Bogatyrëv occupa un poste d'assistant du semestre d'été 1931 au semestre d'hiver 1933/1934.

4 À côté du livre sur les fonctions du costume traditionnel en Moravie slovaque (1937), les textes de Bogatyrëv sur le théâtre de marionnettes et plus largement sur le théâtre populaire ont retenu depuis les années 1970 l'attention des sémioticiens et des théoriciens du théâtre ${ }^{6}$ qui y voient une application des théories sémiotiques de Saussure à la culture contemporaine, ouvrant la voie à la fois à l'étude des structures verbales dans la représentation théatrale et à l'étude de la fonction des objets, cette dernière ayant permis ensuite le développement de la théorie des «performing objects » qui se situe entre sémiotique, ethnologie, anthropologie théâtrale et création contemporaine ${ }^{7}$.

Je voudrais revenir sur ces articles consacrés au théâtre de marionnettes et montrer combien ils furent le fruit d'une situation exceptionnelle à laquelle Bogatyrëv sut être sensible et à laquelle il contribua par la suite lui-même. Intéressé par le théâtre populaire dès ses débuts en Russie ${ }^{8}$, Bogatyrëv fut frappé en étant confronté au théâtre de marionnettes tchèque et à l'intense activité artistique et théorique qu'il suscitait, par les similitudes et les différences et choisit par conséquent de traiter les deux en commun dans une analyse contrastive qui peut rappeler, dans son principe, celle que Roman Jakobson consacra à la poésie dans son livre $\mathrm{Du}$ vers tchèque, principalement dans sa comparaison avec le vers russe (1923). À cette conjonction s'ajoutait aussi, ne serait-ce qu'à travers les historiens et les théoriciens tchèques du théâtre de marionnettes auxquels Bogatyrëv eut recours pour son travail, le contact avec les héritiers de l'esthétique néoherbartienne tchèque, attentive aux caractéristiques concrètes du matériau artistique, dont l'approche interagissait chez Bogatyrëv avec celles des traditions ethnographiques russes et du formalisme tel qu'il le connaissait à travers le Cercle linguistique de Moscou et à travers ses contacts avec certains des membres de l'OPOJAZ. Mais ces années furent marquées également par l'inspiration reçue de la Volkskunde allemande de l'époque en même temps que par la critique lucide de certains de ses aspects, et par l'intérêt pour les travaux de la linguistique et de l'ethnologie française (Meillet, Lévy-Bruhl, Dumézil) ne serait-ce qu'à travers le Centre Ernest-Denis, Centre français de Prague, ses conférences et sa riche bibliothèque ${ }^{9}$.

6 Je commencerai par m'intéresser à ce que les articles de Bogatyrëv nous révèlent de sa fréquentation des théâtres de marionnettes tchèques et de ses réactions à l'effervescence étonnante qu'ils suscitent à l'époque, liée notamment aux débuts de la jeune République tchécoslovaque, avant de passer aux grandes lignes de son analyse : la dignité esthétique du théâtre de marionnettes, illustration d'une poétique du quotidien, les figures de style auxquelles ce théâtre a recours et qui sont analysées dans la lignée des formalistes, la question de l'interaction avec le public qui rejaillit sur les pièces elles-mêmes et leur mise en scène. De manière générale le déplacement de l'analyse sur la représentation 
elle-même en tant que performance ponctuelle, lieu d'échange et entité sémiotique complexe, participe chez Bogatyrëv d'une tendance plus générale à défendre l'« actualité » des pratiques culturelles contre la «survivance», une tendance soustendue par l'idée que ces pratiques ne prennent leur sens qu'à l'intérieur d'un contexte présent donné.

7 Tendue entre deux traditions populaires (le théâtre populaire russe et le théâtre de marionnettes tchèque) et entre les avant-gardes de ces deux mêmes pays, l'étude de Bogatyrëv inclut une réflexion sur les relations entre tradition, improvisation et création, les échanges entre art des élites et art populaire, l'interaction entre le créateur et son public et ouvre sur une analyse de la spécificité du signe théâtral.

\section{Bogatyrëv et l'âge d'or du théâtre de marionnettes en Tchécoslovaquie dans les années 1920 et 1930}

8 Tout touriste passé par Prague sait que la capitale de la Bohême possède une tradition très riche en matière de théâtre de marionnettes, un domaine où les Tchèques ont excellé et excellent encore, tout comme dans l'illustration de livres, le dessin animé, la photographie. Cette tradition remonte au Moyen Âge mais connut une modernisation et un développement considérables durant la seconde moitié $\mathrm{du}$ xviII siècle, qui vit l'émergence de grandes lignées, ou familles, de marionnettistes. Au début du XIX siècle, environ deux cents marionnettistes itinérants parcouraient le pays ${ }^{10}$. L'un d'entre eux surtout, Matěj Kopecký (1775-1847), fut adulé après sa mort comme héros de la renaissance nationale tchèque. Comme le rappelle Bogatyrëv dans son livre, les marionnettistes, en refusant d'obéir aux injonctions des autorités et en continuant à utiliser la langue tchèque, jouèrent un rôle important pendant le renouveau nationaliste du XIX ${ }^{e}$ siècle et dans la propagande de la langue natale en Bohême ${ }^{11}$. Il connaît le rôle particulier accordé dans ce contexte à Matěj Kopecký, auquel il a consacré un article à part entière ${ }^{12}$, mais n'en souligne pas moins la part mythique qu'il y a dans cette héroïsation.

9 Kopecký incarne le type même du marionnettiste populaire de cette époque qui, travaillant dans les zones rurales fortement influencées par les traditions de la culture artistique baroque, contribua à développer des formes théâtrales compatibles avec cette tradition. Tout en restant fidèles au style baroque et en utilisant dans leur dramaturgie les marionnettes pour accentuer la nature symbolique du théâtre, ces marionnettistes populaires développèrent aussi de nouveaux répertoires ${ }^{13}$, familiarisant peu à peu leur audience avec le théâtre de leur époque qui promouvait les idées des Lumières et la renaissance nationale. La mort de Matěj Kopecký en 1847 marqua la fin de cette grande période des marionnettistes populaires tchèques du $\mathrm{XIX}^{\mathrm{e}}$ siècle et vers 1900 le développement $d u$ théatre de marionnettes populaire s'arrêta momentanément. Sa stagnation était due aussi à un changement de style général dans les arts et le théâtre avec l'avènement du réalisme ${ }^{14}$.

10 C'est l'apparition des marionnettistes amateurs dans les milieux aristocratiques et dans le cercle familial qui permit un renouveau du théâtre de marionnettes et contribua en 1902 à la naissance à Prague d'un club du théâtre de marionnettes qui possédait une scène propre. Dès 1903, les membres de ce club organisèrent la première rencontre des amis du théâtre de marionnettes et préparèrent pour cette occasion une mise en scène de l'opéra 
de Smetana La fiancée vendue pour montrer toutes les possibilités offertes par ce type de théâtre. L'année suivante, une deuxième rencontre eut lieu à Plzeň et en 1905 des marionnettistes de Kladno organisèrent la première compétition internationale de théâtre de marionnettes. En 1911, enfin, fut organisée à Prague une importante exposition de marionnettes qui rencontra un grand succès auprès du public. Ce fut l'un des premiers événements par lesquels le publiciste Jindřich Veselý (1887-1939) chercha à soutenir le développement du théâtre de marionnettes. La même année fut créée l'association tchèque des amis du théâtre de marionnettes qui publia à partir de 1912 le premier magazine au monde spécialisé dans ce domaine, Loutkár (plus tard Český Loutkár̆, Le marionnettiste tchèque), dont Jindřich Veselý devint l'éditeur ${ }^{15}$. Il faut noter ici que les recherches réalisées par Jindřich Veselý sur l'histoire et l'actualité du théâtre de marionnettes, sur ses personnages, son répertoire et ses aspects matériels et techniques, ont été la source principale de Bogatyrëv pour l'étude du théâtre de marionnettes tchèque et qu'il le cite pratiquement à chaque page de son livre.

11 À côté de Prague, Plzeň devint un autre centre important pour les activités liées au théâtre de marionnettes. Un théâtre de marionnettes y existait depuis 1902, mais une nouvelle ère commença en 1913 lorsque toutes les compagnies existantes s'unirent sous la houlette d'une organisation charitable organisant des séjours de vacances pour les enfants de milieux défavorisés. Le développement du "Théâtre de marionnettes des Campeurs » fut considérablement influencé par la présence et le professionnalisme du marionnettiste populaire Karel Novák (1862-1940), qui devint membre honoraire du théâtre. C'est grâce à sa coopération avec les membres amateurs de la troupe, investis surtout dans le travail dramaturgique, que le profil individuel des "Campeurs" prit forme. Le dessinateur Josef Skupa (1892-1957) rejoignit le groupe en 1917, il commença à mettre en pratique ses idées sur le théâtre de marionnette moderne et devint bientôt la personnalité centrale de la troupe. Skupa pensait que le théâtre de marionnettes pouvait s'adresser également au grand public et durant les dernières années de la guerre, alors que la censure s'était durcie, il profita du désintérêt des censeurs pour les scènes de marionnettes et organisa des représentations en soirée pour adultes. Ces représentations de cabaret sur la scène du Théâtre des Campeurs firent sensation en raison de leur actualité politique : durant les derniers mois de la guerre, un Kašpárek ${ }^{16}$ révolutionnaire y enterrait symboliquement l'Empire austro-hongrois sur la scène ${ }^{17}$. Dans son livre Bogatyrëv évoque le théâtre de Plzeň comme le plus grand théâtre de marionnettes tchèque et signale qu'il a eu la chance d'assister en coulisse à une de ses représentations. Il décrit comment, lorsqu'un virtuose comme Karel Novák prend une marionnette dans ses mains, celle-ci paraît s'animer immédiatement ${ }^{18}$.

12 Après la guerre, de nouveaux théâtres apparurent chaque année. Ils jouaient régulièrement le samedi et le dimanche pour les jeunes spectateurs. À la fin des années 1930 il y en avait trois mille, situés aussi bien dans les grandes villes (20-35 groupes étaient actifs à Prague), que dans de petits villages. Comme les marionnettistes trouvèrent progressivement des locaux permanents dans des écoles, des bibliothèques, des salles municipales et surtout dans les centres Sokol ${ }^{19}$, ils commencèrent à remplacer les scènes simples et mobiles par des constructions scéniques plus complexes, dotées d'équipements modernes pour la lumière et le son permettant une mise en scène plus élaborée, et améliorèrent l'utilisation de l'espace scénique. Ceci constitua le couronnement d'une série de réformes qui libérèrent progressivement la scène des bordures superflues, restes du système de décoration baroque limitant les mouvements des marionnettes. Ce boom aboutit même à la construction de bâtiments 
spécialisés. En 1923, Bogatyrëv comptabilise dans son livre à peu près mille théâtres de marionnettes populaires et itinérants et deux mille scènes liées à des écoles ou à des organismes culturels; il note qu'il faut y ajouter évidemment un nombre incalculable de théâtres familiaux ${ }^{20}$.

Un des traits caractéristiques de toute la période est la participation exceptionnelle et continue de sculpteurs, d'artistes, de dessinateurs et d'enseignants des écoles artistiques à la direction d'un grand nombre de ces troupes, une participation due en bonne partie au prestige atteint à l'époque par le théâtre de marionnettes en tant que système artistique. Le phénomène le plus important dans cette expansion après la Première Guerre mondiale, fut l'établissement de plusieurs théâtres de marionnettes permanents dont le programme incluait des buts artistiques précis. À travers ceux-ci ces théâtres souhaitaient se différencier des troupes plus populaires avec des standards artistiques moindres. Le cercle de ces théâtres proposant des représentations régulières s'étendit audelà du Théâtre des Campeurs de Plzeň (1913-36), qui poursuivait ses activités d'avantguerre, et inclut désormais Prague où fonctionnaient le Théâtre de marionnettes de l'éducation artistique (1914-1954) et le Royaume des marionnettes (de 1920 à nos jours). Ils furent également rejoints par le Théâtre de marionnettes Sokol de Prague-Libeč (1922-1939) et par plusieurs autres théâtres des alentours de Prague, par exemple le Théâtre de marionnettes de Turnov (1922-1939) dirigé par le peintre Karel Vik, le Théâtre de marionnettes Sokol de Liberec (1925-1938) et d'autres. Un des moments artistiques forts de cette période est constitué par la tentative de Skupa de remplacer le vieux Kašpárek, jugé archaïque. Ces efforts aboutirent après 1920 à la création des deux personnages de Spejbl et Hurvínek ${ }^{21}$ qui connurent rapidement les faveurs du public ${ }^{22}$.

En 1929, dans le cadre du "Royaume des marionnettes », une exposition internationale de marionnettes fut organisée à Prague. Bogatyrëv consacra un compte rendu aux marionnettes russes présentées dans ce contexte dans les revues Central'naja Evropa et Loutkáŕr3. C'est à cette occasion que fut fondée l'UNIMA, l'Union internationale des marionnettes, dont Jindřich Veselý fut élu président. Dans les années 1930, plusieurs jeunes directeurs de théâtres de marionnettes cherchèrent à trouver de nouveaux styles et mirent en scène des œuvres exigeantes dotées d'une forte tradition, non pas simplement dans le but de rendre le répertoire plus attrayant, mais aussi parce qu'ils souhaitaient pour ainsi dire tester leurs capacités. Au Sokol de Prague on produisit par exemple Faust de Goethe et Hamlet de Shakespeare ${ }^{24}$.

Fidèle à son approche fonctionnaliste, Bogatyrëv s'intéresse aussi dans son livre aux buts poursuivis par le théâtre de marionnettes et analyse la manière dont il est utilisé comme outil pédagogique dans des mises en scène de contes populaires mais aussi de pièces classiques (Électre, Hamlet) faites par des élèves. Il rapporte avoir vu dans une école une représentation d'Électre avec des marionnettes. La scène était une réplique exacte d'une scène grecque antique et la représentation avait été organisée avec l'aide d'un professeur d'université qui était l'un des meilleurs connaisseurs du théâtre classique. Il évoque également, avec moins d'enthousiasme cependant, comment le théâtre de marionnettes est utilisé aussi pour la propagande médicale dans des saynètes où les marionnettes représentent des microbes ${ }^{25}$.

16 Parmi les articles de Bogatyrëv sur le théâtre de marionnettes, un seul n'est pas consacré aux marionnettes tchèques. Il s'agit de l'article sur les marionnettes de Münster ${ }^{26}$ qui commence par une description assez littéraire de la foire traditionnelle de Münster, le send, où Bogatyrëv a assisté également à des représentations de marionnettes. Il 
enchaîne en faisant référence, sans véritable transition, à la théorie de la " dégradation du patrimoine culturel » ou, peut-être plus justement, du " patrimoine culturel tombé dans le domaine populaire " (gesunkenes Kulturgut) du germaniste Hans Naumann (1886-1951), et en rappelant l'urgence du travail de collecte et de conservation. Puis Bogatyrëv raconte comment, pendant la durée de la foire, il a assisté tous les jours aux représentations du théâtre de marionnettes présent sur la foire, observant à la fois la scène et le public. Il a également été invité à une représentation exceptionnelle, non abrégée, du spectacle, donnée dans un hospice de la ville, lors de laquelle les onze actes furent joués sans coupures et dont la durée fut de deux heures, pause incluse. Il commence par une liste des personnages et une description de leurs caractéristiques physiques et vestimentaires (Kasper (Guignol), le Schupo (Gendarme), le Chien, le Nègre, deux Hussards, le Sultan turc, le Russe, le Diable, un voleur, sa femme, un Monsieur, la Mort, et Kasper vieux), et ajoute qu'on retrouve des figures semblables dans le théâtre de marionnettes russe. Mais contrairement à la manière dont se termine traditionnellement ce type d'histoire en Russie (le Diable ou le Chien emportent Kasper, ou son équivalent russe, en enfer), nous avons ici, comme en Tchécoslovaquie, la victoire de Kasper. Tous les personnages parlent la langue allemande courante avec quelques mots de dialecte. La langue du Russe exige une analyse spécifique : il parle sans articles, dit non pas «à moi » ou « à toi » mais « à soi » (sebja), prononce le « $\mathrm{o}$ » d'une manière proche du « $\mathrm{a}$ ». Tout ceci paraît à Bogatyrëv une imitation correcte et il souligne combien lui semble intéressante l'utilisation des langues des minorités dans le théatre de marionnettes. Il signale en passant l'hypothèse que le théâtre de marionnettes a voyagé d'Allemagne en Russie. L'article se termine par quelques éléments sur l'histoire de ce théâtre et de cette troupe spécifiques, tels qu'il a pu les reconstituer à partir des récits du marionnettiste et de sa femme. Les origines en remontaient apparemment à l'époque des guerres napoléoniennes. Difficile, cependant, de dire de quelle époque date la pièce jouée durant cette foire. Vu les manuscrits disponibles en Tchécoslovaquie, Bogatyrëv doute toutefois que les pièces ne soient jamais couchées par écrit, comme le lui a dit le marionnettiste.

Comme nous l'avons vu, ces articles sont essentiellement consacrés à l'actualité du théâtre de marionnettes. C'est dans le livre de 1923 que Bogatyrëv a développé sa réflexion théorique sur laquelle je vais à présent m'attarder.

\section{La dignité esthétique du théâtre de marionnettes}

Bogatyrev n'est évidemment pas le premier à s'intéresser au théâtre de marionnettes et il signale au début de son ouvrage ${ }^{27}$ les faveurs que ce dernier a rencontrées chez une longue série d'auteurs, depuis Platon jusqu'à Béranger en passant par Shakespeare. Il sait bien par ailleurs qu'un certain nombre d'auteurs dramatiques et de metteurs en scène modernes ont trouvé une inspiration non négligeable dans le théâtre de marionnettes. Du côté russe, il cite Nikolaj Evreinov, Vsevolod Meyerhold et Aleksandr Tairov, du côté anglais Bernard Shaw et le théoricien du thêâtre Edward Gordon Craig, sans doute l'un des plus grands spécialistes du théâtre de marionnettes à l'époque ${ }^{28}$. Il considère néanmoins tout au long de l'ouvrage que la dignité esthétique du théâtre de marionnettes n'a pas été suffisamment reconnue dans les études littéraires, alors que, de par sa particularité, il apporte selon lui des éclaircissements importants concernant l'esthétique théâtrale en général. L'originalité de son étude vient principalement de la conjonction d'une approche littéraire et esthétique avec un corpus relevant, aux yeux 
des classifications usuelles, de l'étude des arts populaires, d'une confrontation entre les méthodes de ces deux domaines scientifiques et des théories alors en cours dans chacun d'entre eux.

Le livre de 1923 se compose de trois parties : 1) description du théâtre de marionnettes tchèque ; 2) description du théâtre populaire russe ; 3) analyse des traits communs aux méthodes stylistiques des deux traditions. L'analyse se fonde sur la comparaison entre les deux pratiques théâtrales. Dans la première partie du chapitre consacré au théâtre de marionnettes tchèque, Bogatyrëv évoque le rôle important de ce dernier dans la vie culturelle tchèque passée et surtout présente. Il décrit les deux principaux types de marionnettes utilisés: les marionnettes enfilées sur la main comme un gant, marionnettes à gaines, et les marionnettes actionnées au moyen de fils. Il note que la première variante est plus rare en Tchécoslovaquie, mais il raconte avoir pu assister à Prague à un spectacle de ce type pendant une foire, dans une pièce regroupant Kašpárek, un Juif, sa Femme et le Diable ${ }^{29}$. Il s'intéresse ensuite aux différents courants artistiques qui marquent le théâtre de marionnettes. Le courant majoritaire est, selon lui, naturaliste. Il est représenté notamment par les marionnettistes populaires qui, grâce à leur virtuosité, parviennent à faire oublier que les marionnettes sont en bois. Comme nous l'avons vu, Bogatyrëv comptait parmi ces virtuoses Karel Novák, qui sut donner son empreinte artistique au théâtre de Plzeň et lui transmettre son savoir technique. À l'intérieur même de cette orientation naturaliste assez générale, certaines conventions non naturalistes se sont maintenues, comme c'est le cas notamment du style déclamatoire conventionnel de différents personnages. Bogatyrëv note qu'il existe cependant désormais une nouvelle mode consistant à styliser beaucoup plus les visages et les corps des marionnettes. Il est sensible ici aux tendances artistiques qui aboutiront notamment aux personnages de Skupa déjà évoqués, Spejbl et Hurvínek, ce dernier étant cité ultérieurement dans l'article sur les marionnettes de Münster ${ }^{30}$. L'utilisation de mouvements maladroits et stylisés augmente selon Bogatyrëv l'expressivité, à un point tel que même des acteurs ne sauraient y parvenir. Il considère pour cette raison que ce style a certains avantages par rapport au style naturaliste et rappelle, au tout début du livre, qu'un certain nombre de dramaturges et de metteurs en scène recommandent la fréquentation des marionnettes pour améliorer le jeu des acteurs ${ }^{31}$.

Bogatyrëv commence ensuite le chapitre sur le théâtre populaire russe, en notant de manière critique que ce dernier n'a pas attiré l'attention des spécialistes de littérature russe, car il leur est esthétiquement étranger et inacceptable ${ }^{32}$. Du côté des spécialistes du folklore, les choses ne se présentent guère mieux selon lui car, qu'ils soient issus de l'école anthropologique, mythologique, intertextuelle ou historique, les chercheurs ne s'intéressent au folklore qu'en tant que survivance d'une antiquité glorieuse. Dans le folklore, ce n'est finalement que cette dernière qui a une quelconque valeur à leurs yeux, le reste est dégénérescence due à l'influence corruptrice de la ville et de l'usine ${ }^{33}$. Or le théâtre populaire russe est précisément un phénomène récent et il n'offre aucune chance d'y trouver des traces de l'antiquité. Même si, selon Bogatyrëv, la distinction entre création collective et création individuelle tend à s'estomper et si l'on reconnaît de plus en plus que l'altération la plus simple faite dans une représentation est déjà à sa manière une création individuelle, il lui semble que le théâtre populaire évolue avec la vie des gens et qu'il est une expression relativement immédiate de leurs besoins les plus pressants et d'une poétique de tous les jours ou poétique du quotidien ${ }^{34}$. Cette insistance sur le caractère actuel des pratiques culturelles vient du rejet d'un certain historisme et 
de l'évolutionnisme qui y est lié, tel qu'il s'affirme au même moment dans un bilan de la philologie russe écrit en commun avec Jakobson ${ }^{35}$ et tel qu'il sera développé de manière plus systématique dans le livre de Bogatyrëv sur les actes magiques, ${ }^{36}$ où l'on retrouve l'idée que les actes magiques ne sont pas intéressants en tant que survivance mais en tant que pratique actuelle remplissant des fonctions bien précises dans un contexte donné37. Mais cette perspective trouve également des échos et un certain soutien dans les orientations émanant de l'École esthétique tchèque qui, chez quelqu'un comme le défenseur d'un «formalisme concret » Otakar Hostinský (1847-1910) par exemple, tente de revaloriser en théorie musicale le moment de la représentation par rapport à une certaine «tyrannie » de la partition, de défendre le mélange des arts ou intermédialité, ainsi que, de manière générale, l'évolution des arts en fonction de contextes donnés et de conventions mouvantes par opposition à toute forme de dogmatisme ${ }^{38}$. Elle donnera lieu, comme nous le verrons plus loin, à un dialogue intéressant entre Bogatyrëv et l'élève de Hostinský, Otakar Zich, qui fera véritablement de l'esthétique théâtrale l'étude d'une « performance ».

Si on a reproché à Bogatyrëv de n'avoir su, malgré les critiques qu'il lui adresse, se libérer totalement de la théorie des survivances dans son livre sur les actes magiques ${ }^{39}$, de même qu'il reste fidèle en fin de compte aux lois de l'anthropologue écossais James George Frazer (1854-1941) (loi de contact et loi de similitude) pour expliquer la « logique » de la magie, il peut sembler que dans le cas du théâtre populaire et du théâtre de marionnettes, précisément en raison de la dimension esthétique qui entre en jeu, il y parvient mieux, atteignant par là un autre niveau d'analyse. C'est parce que, au nom d'une poétique du quotidien, Bogatyrëv prend le théâtre de marionnettes " au sérieux » du point de vue esthétique, qu'il peut ensuite lui appliquer les outils d'analyse réservés habituellement à l'analyse de textes littéraires plus élaborés, comme nous allons le voir maintenant.

\section{Théâtre de marionnettes, théâtre populaire et figures de style}

Le troisième chapitre du livre de Bogatyrëv est consacré aux traits communs des deux pratiques théâtrales dans leurs usages stylistiques. C'est sans doute ici que l'héritage de certains travaux formalistes russes est le plus net.

Bogatyrëv examine les styles de discours des caractères comiques dans le théâtre de marionnettes tchèque (surtout chez Kašpárek et chez les paysans) et les compare avec ceux du théâtre russe. Il y relève, et illustre avec un nombre très important d'exemples détaillés et de citations, l'utilisation d'oxymores, de métathèses (transposition des parties d'un mot ou d'une phrase l'une à la place de l'autre), le jeu avec les synonymes et les homonymes, le jeu de la métaphore et notamment l'effet comique créé par la métaphore prise à la lettre ou la réalisation de la métaphore, l'accumulation des répétitions ${ }^{40}$. Il montre, enfin, comment des scènes entières sont construites sur des contrastes, par exemple lorsque des paroles sont prononcées avec un effet dramatique ("Adieu ma chère Cécile») puis répétées avec une légère différence, un léger décalage, déclenchant un effet comique (« Adieu ma Thérèse aux grandes dents »). Bogatyrëv note également l'utilisation du non-sens. Tout aussi importantes lui paraissent les bizarreries caractéristiques du langage de certains personnages (changements phonétiques, morphologiques et lexicographiques). Il renvoie sur ce point aux observations faites par 
Veselý sur le langage des personnages représentatifs des élites. Ceux-ci se singularisent dans le théâtre de marionnettes par une langue tchèque " dégradée ", caractérisée par des germanismes, un mélange de mots étrangers et de mots tchèques, une grammaire terrible, surtout dans les scènes passionnées qui en deviennent d'autant plus comiques ${ }^{41}$. Il s'agit là de quelque chose qui selon Bogatyrëv n'existe pas dans le théatre populaire russe, où ce sont surtout les langues des minorités qui sont utilisées pour créer un effet comique. Ce dernier phénomène se retrouve également en pays tchèque, où les juifs par exemple sont caricaturés à travers leur prononciation germanique des consonnes ${ }^{42}$.

Bogatyrëv en arrive au répertoire de ces deux formes de théâtre. Jusqu'à présent il a analysé le théâtre de marionnettes stylistiquement comme n'importe quel texte littéraire. Il veut à présent dire quelques mots sur les spécificités théâtrales du théâtre de marionnettes tchèque et du théâtre populaire russe. Il note tout d'abord pour tous deux la grande variété du répertoire. Ainsi parmi les cinquante et une pièces publiées par le fils du célèbre marionnettiste Matěj Kopecký, on trouve aussi bien le vieux drame médiéval Doktor Faustus que des dramatisations de vieilles légendes historiques. Parmi ces dernières, les plus populaires sont les histoires de chevaliers. Comme Bogatyrëv le note, en se référant de manière assez surprenante à une remarque du " philosophe russe, le Prince Sergej Trubeckoj ", c'est-à-dire sans doute à Sergej Nikolaevič Trubeckoj (1862-1905), connu pour sa théorie de l'essence collective de la nature humaine, le public a toujours eu un goût très prononcé pour les sujets historiques, d'une part à cause de l'attirance, paradoxale en apparence seulement, des gens simples pour les histoires liées à la cour et au monde aristocratique, d'autre part à cause du faste des costumes ${ }^{43}$.

Pour ce qui est de la composition des pièces, le théâtre de marionnettes tchèque dépend des personnages qui font partie du théâtre urbain tchèque vivant et il $\mathrm{y}$ a de manière générale de nombreux passages de l'un à l'autre ${ }^{44}$. Ce n'est pas le cas selon Bogatyrëv dans le théâtre populaire russe qui est presque sans lien aucun avec le théâtre des couches aisées car il a suivi une voie propre et a été obligé de développer ses propres méthodes. Cette particularité du théâtre de marionnettes tchèque est très intéressante, puisqu'elle permet d'étudier les changements que les pièces du répertoire urbain subissent quand elles sont reprises par des marionnettistes en contact avec le public populaire. Bogatyrëv insiste pour finir sur certaines particularités techniques du théâtre de marionnettes, comme le fait qu'il existe souvent un seul acteur pour plusieurs personnages. En ce qui concerne les costumes, il souligne, comme Veselý auquel il se réfère de nouveau ici, que les costumes du théâtre de marionnettes n'obéissent pas à la vraisemblance historique mais sont très fortement stylisés.

Dans toutes ces considérations, Bogatyrëv attache évidemment une grande importance au goût du public, mais il va plus loin ensuite, cherchant à montrer dans la dernière partie de son livre comment le public participe directement à la représentation.

\section{La question du public}

27 Bogatyrëv regrette que la théorie du théâtre ne soit pas, de manière générale, assez attentive au public. Il signale qu'il a trouvé dans la revue Loutkár une série d'observations très intéressantes faites sur un public d'enfants dans un théâtre de marionnettes et sur les modalités de sa participation. En ce qui concerne le théâtre populaire russe, il estime qu'il n'existe dans son cas pratiquement pas d'études sur cette question, alors que le public y fait souvent fonction de chœur dans les représentations. Il 
faut signaler ici que l'année même de la parution du livre de Bogatyrëv, celle du livre de l'angliciste allemand Ludwig Schücking, Die Soziologie der literarischen Geschmacksbildung [La sociologie de la formation du goût littéraire], est un événement.

Bogatyrëv poursuit par une "analyse formaliste» des ressorts linguistiques des personnages comiques dans le théâtre de marionnettes tchèque, selon l'expression qu'il utilise lui-même pour qualifier son travail, et montre l'abondance de ces derniers et leur différence, ou spécificité, par rapport à ceux de la comédie contemporaine. La comparaison avec le théâtre populaire russe, au contraire, a fait apparaître beaucoup de points communs qui ne sauraient s'expliquer par des emprunts ou des influences mais par une unité des goûts et des demandes du public dans les deux pays ${ }^{45}$.

Dans l'article sur les marionnettes à Münster, Bogatyrëv commente longuement la technique de l'adresse au public. Les questions que Kasper adresse incessamment au public, invité ainsi à collaborer à la pièce, lui en paraissent un des ressorts artistiques les plus importants. Certaines scènes ne sont même pas imaginables sans cette interaction et cette réciprocité. On trouve ces échanges avec le public aussi dans le théâtre de marionnettes russe ${ }^{46}$. Si les pièces russes commencent presque toujours par un dialogue entre Petruška et le public, on trouve quelque chose de semblable dans le théâtre de marionnettes de Plzeň avec le personnage de Hurvínek. Une pratique apparentée, fréquente dans les théâtres populaires de Russie, d'Angleterre ou du Turkestan, est celle du dialogue entre marionnettes et musiciens. À Münster aussi les dialogues avec le public sont très développés, comme le note Bogatyrëv, et remplissent parfois des scènes entières $^{47}$. De là, il n'y a qu'un pas pour lui à l'introduction d'acteurs vivants directement dans la salle, comme c'est le cas par exemple au Théâtre d'État pour enfants de SaintPétersbourg, un aspect qu'il a traité également dans un de ses articles sur les marionnettes russes exposées à Prague ${ }^{48}$.

Cette question se relie à celle de l'improvisation omniprésente dans tout théâtre populaire, où elle donne lieu à des ajouts ou au contraire à des retraits qui suivent au plus près le goût et les réactions immédiates du public. Parfois ce sont même certaines scènes extraites d'une pièce qui sont ainsi insérées dans une autre pièce, pour la simple raison qu'elles rencontrent une faveur particulière auprès du public ${ }^{49}$. De manière générale, Bogatyrëv tient à prendre ses distances par rapport à la tendance héritée du romantisme consistant à séparer strictement les traditions orales, issues d'une création collective, et la littérature, considérée comme création individuelle ${ }^{50}$. Comme nous l'avons vu précédemment, il voit à cette époque en toute altération opérée par un exécutant sur un modèle transmis, une forme de création, rejoignant donc ici la position défendue au tournant du siècle par le spécialiste autrichien des chansons populaires Josef Pommer (1845-1918) et qualifiée de «théorie de la production» (Produktionstheorie, le peuple n'est pas passif, il produit vraiment l'art populaire).

31 Cette question sera reprise et réexaminée dans la contribution présentée par Bogatyrëv et Jakobson au Congrès international de linguistique de La Haye de 1928, «Le folklore comme forme particulière de création ", dans laquelle les deux amis maintenaient cette fois-ci, en s'appuyant sur la distinction saussurienne entre langue et parole qui est un des axes centraux de leur démonstration, l'idée d'un type de création spécifique qui serait celui du folklore. Le texte se présente d'ailleurs lui-même comme une tentative pour définir les " propriétés distinctives de la créativité folklorique associées au caractère des interrelations entre le narrateur et le corps collectif ${ }^{\prime 51}$. La question du public y joue un rôle central puisque l'analyse montre que le folklore ne conserve que les formes qui ont 
fait leurs preuves d'un point de vue fonctionnel pour une collectivité donnée. Si une forme perd toute fonction, elle dépérit et disparait. À la différence de la littérature, il existe dans le folklore, qui est oral, une censure préventive.

Par opposition à ce que Bogatyrëv a pu écrire dans son livre sur le théâtre de marionnettes, les deux amis taxent ici de réalisme naïf les spécialistes du folklore, tout particulièrement slaves, qui partent de l'idée qu'il n'y a pas de différence principielle entre la poésie orale et la littérature, alors que cette dernière est le produit d'une création individuelle. Dans le folklore le rapport entre l'œuvre d'art et son objectivation (c'est-à-dire les variantes) est analogue au rapport entre langue et parole tel que le définit Saussure. Comme la langue, l'œuvre du folklore est extra-personnelle et ne vit qu'une existence potentielle. Elle n'est qu'un complexe de normes et d'impulsions, le canevas d'une tradition actuelle que le récitant fait revivre à travers les ornementations de la création individuelle. Le folklore est donc réglé sur la langue, la littérature sur la parole. Prenant position cette fois-ci, implicitement, contre la " théorie de la réception " (Rezeptionstheorie) du spécialiste allemand des chansons populaires John Meier (1864-1953), les deux amis soulignent que la thèse "le peuple ne produit pas, il reproduit " perd ainsi sa pertinence parce qu'il n'est pas légitime de poser une frontière indépassable entre la production et la reproduction et de considérer cette dernière comme inférieure. La reproduction n'est pas reprise passive, elle est aussi un acte de création qui se révèle dans le choix des œuvres reprises, dans leur réaménagement pour d'autres habitudes et exigences.

De manière générale, cet intérêt pour le public, ou pour les phénomènes de réception, et la prise de conscience de la nécessité de les intégrer à l'analyse, constitue un des horizons $\mathrm{du}$ formalisme, comme théorie esthétique et comme approche des phénomènes artistiques. Si les formalistes du XIX ${ }^{\mathrm{e}}$ siècle ont effectivement pu être critiqués pour une approche trop immanente, réduisant les formes à des relations entre des éléments constitutifs, et incapable de les inscrire dans des dynamiques autres qu'internes, le début $\mathrm{du} \mathrm{Xx}^{\mathrm{e}}$ siècle voit paraître toute une série de tentatives pour intégrer notamment la dimension de la réception. C'est ce que montre entre autres le cas d'Otakar Zich (1879-1934), théoricien de l'art et compositeur tchèque, héritier du formalisme néoherbartien, qui critiquait déjà l'esthétique formelle dans son essai sur la perception esthétique de la musique (Estetické vnímání hudby. Psychologický rozbor, 1911 [La perception esthétique de la musique : analyse psychologique]) parce qu'elle ne parvenait pas, selon lui, à tenir compte du fait que ce n'est pas la forme pure qui plait mais seulement la forme expressive et signifiante. L'analyse ne peut donc se contenter des relations formelles entre les parties puisque ces dernières n'acquièrent leur fonction esthétique et signifiante qu'en relation avec le tout qui sera saisi dans une analyse sémiotique de la structure de l'œuvre ${ }^{52}$. Dans son Esthétique de l'art dramatique (Estetika dramatického umění, 1931), Zich développe une analyse de la représentation théatrale comme " performance " qui implique précisément la collaboration du spectateur et donc, au niveau de l'analyse, la prise en compte des phénomènes de réception ${ }^{53}$. Bogatyrëv a publié ses textes sur le théâtre de marionnettes dans les années 1920 et au tout début des années 1930, il ne pouvait guère s'y référer au livre de Zich, mais il se livra à une réflexion critique sur ce dernier dans deux textes ultérieurs: "Les signes du théâtre (1938) et "Le théâtre populaire tchèque et slovaque " $(1940)^{54}$. Là où Zich s'appuie essentiellement dans ses analyses sur le drame réaliste, il semble à Bogatyrëv que le théâtre populaire permet de mieux comprendre la transformation comme procédé central du théatre, puisque tout ce 
qui se trouve sur la scène est, à l'image de l'acteur qui est perçu par le spectateur à la fois comme personne vivante et comme système de signes visuels et auditifs, d'une nature double. Cette double nature, mise en avant également par Zich, est précisément mieux rendue selon Bogatyrëv par le théâtre populaire que par le théâtre réaliste. Tout ce qui précède tend à montrer que le théatre de marionnettes, avec ses tendances non- ou antiillusionnistes et ce qu'il révèle de l'interchangeabilité de l'acteur humain et de l'objet, même si le rôle de la voix humaine reste indispensable, a joué lui aussi un rôle important dans l'émergence d'une analyse sémiotique du théâtre.

\section{Conclusion} relativement simple et dépourvu de thèses fortes. Mais la perspective de Bogatyrëv, mêlant ethnographie et esthétique, est différente de celle des folkloristes traditionnels. D'un autre côté, dans sa banalité apparente, l'idée d'un besoin de poésie et d'une poétique du quotidien, issue du contact étroit avec les arts populaires, introduit aussi une dimension anthropologique qui manque parfois à certains des développements les plus littéraires et les plus techniques du structuralisme. Elle permet également à Bogatyrëv d'insister sur les points communs entre les formes artistiques les plus frustres et les formes plus élaborées, en montrant qu'il y a des échanges entre les deux, non pas seulement dans le sens culture élaborée vers culture populaire, comme le montrait la théorie des survivances (la culture populaire se nourrirait des « restes»), mais aussi dans l'autre sens.

On ne saisira sans doute toute la signification et la finesse du travail de Bogatyrëv et de sa sensibilité artistique que si on rapporte ces réflexions sur le théâtre de marionnettes à d'autres figures qui l'ont intéressé à l'époque et qui entretenaient elles-mêmes une série de riches correspondances avec le théâtre de marionnettes et les traditions du théâtre populaire. Il faut rappeler ici d'une part que Bogatyrëv a traduit en russe Le brave soldat Chvéik de Jaroslav Hašek (paru entre 1921 et 1923) et, d'autre part, qu'il a participé avec deux articles au recueil sur Chaplin publié par Šklovskij et d'autres en $1923^{55}$, la même année donc où paraît le livre sur le théâtre de marionnettes.

Ces exemples permettent de comprendre que ce qui intéresse aussi Bogatyrëv dans le théâtre de marionnettes, ce sont les perspectives qu'il ouvre sur la modernité artistique et sur la création contemporaine. Si le théâtre de marionnettes est marqué à l'époque en Tchécoslovaquie par un important investissement artistique, la scène tchèque moderniste connaît également au même moment une série d'échanges improbables, ou au contraire très probables, entre le théâtre populaire et l'avant-garde la plus expérimentale. Bogatyrëv lui-même contribua à cette ouverture comme le montre l'inspiration que reçurent de lui le protéiforme et dynamique Emil František Burian (1904-1959) et son Théâtre D 34. Les réflexions de Bogatyrëv sur le folklore incitèrent Burian à présenter des pièces du répertoire populaire dans son théâtre notamment avec les deux Suites populaires (Lidová suita) de 1938 et de $1939^{56}$. Le dépassement des frontières nationales qui caractérise ces rencontres praguoises s'accompagne ainsi également d'un dépassement des séparations entre, d'un côté, formes artistiques populaires et apparemment "archaïques» et, de l'autre, formes artistiques urbaines et avantgardistes, entre discours théorique et pratique artistique. 


\section{NOTES}

1. Pëtr Bogatyrëv, Češskij kukol'nyj $i$ russkij narodnyj teatr [Le théâtre de marionnettes tchèque et le théâtre populaire russe], Sborniki po teorii poètičeskogo jazyka [Recueil sur la théorie de la langue poétique] VI, Berlin: Opojaz, 1923. Il existe une traduction anglaise (avec quelques coupures) : Pyotr Bogatyrëv, « Czech Puppet Theatre and Russian Folk Theatre », The Drama Review 43 (3), 1999, p. 97 ; et une traduction italienne, Petr Bogatyrev, Il teatro delle marionette, traduction de Maria Di Salvo, Brescia : Grafo, 1980.

2. Pëtr Bogatyrëv, "Češskij kukol'nyj teatr " [Le théâtre de marionnettes tchèque], Central'naja Evropa 1 (3), 1927, p. 7-9 ; « Matěj Kopecký », Central'naja Evropa 1 (25), 1927, p. 9-10 ; « S"ezd dejatelej kukól'nogo teatra v Prage » [Le Congrès des amis du théâtre de marionnettes à Prague], Central'naja Evropa 3(5), 1929, p. 8-9; «Vystavka kukol'nogo teatra SSSR v Prage » [L'exposition du théâtre de marionnettes de l'Union soviétique à Prague], Central'naja Evropa 3 (20), 1929, p. 5-8.

3. Pëtr Bogatyrëv, « Ruské loutkové divadlo » [Le théâtre de marionnettes russe], Loutkár 7(9), 1922/1923, p. 64-65 ; "Výstava ruského loutkářství v Praze» [L'exposition de marionnettes russes à Prague], Loutkáŕ 16, 1929/1930, p. 43-48.

4. « Le théâtre russe de marionnettes (à l'occasion du Congrès des amis des marionnettes à Liège (22/10/1930)) ", Loutkáŕ 17 (6), 1930/1931, p. 160 ; et 17 (7), 1930/1931, p. 184.

5. Pëtr Bogatyrëv, "Puppentheater in Münster», Prager Presse, 11 (227), 23/09/1931, Dichtung und Wahrheit Beilage 34, p. I-III, Reprint : Zeitschrift für Volkskunde 94 (1), 1998, p. 1-5.

6. Voir Ladislav Matejka, Irwin R. Titunik, éds., Semiotics of Art: Prague School Contributions, Cambridge, MA, - Londres: The MIT Press, 1976 (avec notamment des textes de Jan Mukařovský, Pëtr Bogatyrëv, Roman Jakobson, Jindřich Honzl, Jiř́ Veltruský, Felix Vodička). Les textes de Bogatyrëv publiés en traduction anglaise dans ce volume sont les suivants : « Kroj jako znak. Funkční a strukturální pojetí v národopisu » [Le costume comme signe. La conception fonctionnelle et structurale en ethnographie], p. 13-19; "La chanson populaire du point de vue fonctionnel», p. 20-32; «Znaky divadelní » [Les signes du théâtre], p. 33-50 ; «Lidové divadlo české a slovenské » [Le théâtre populaire tchèque et slovaque], p. 51-56. Signalons que le troisième de ces textes fut publié en traduction française au début des années 1971 dans la revue Poétique: P. Bogatyrëv, «Les signes du théâtre », Poétique, 8, 1971, p. 517-530.

7. Voir notamment Frank Proschan, «The Semiotic Study of Puppets, Masks, and Performing Objects from Semiotic Perspectives », Semiotica 43 (1-4), 1983, p. 3-44; et, dans le même numéro que le texte de Bogatyrëv sur le théâtre de marionnettes: John Bell, «Puppets, Masks, and Performing Objects at the End of the Century », The Drama Review 43 (3), 1999, p. 15-27.

8. Roman Jakobson raconte dans ses souvenirs concernant Bogatyrëv que ce dernier vouait une véritable passion au théâtre, fréquenta assidûment les scènes moscovites en tant que lycéen puis étudiant et envisagea un temps de devenir acteur. Voir Roman Jakobson, «Pëtr Bogatyrëv (29.I.93-18.VIII.71). Expert in Transfiguration», in Ladislav Matejka, éd., Sound, Sign and Meaning: Quinquagenary of the Prague Linguistic Circle, University of Michigan Press, 1978, p. 29-39. 
9. Voir Annie Guénard, «Les instituts français en Europe centrale et orientale dans les années 30 ", in Maria Delaperrière, Antoine Marès, éds., Paris "capitale culturelle " de l'Europe centrale? Les échanges intellectuels entre la France et les pays de l'Europe médiane 1918-1939, P. : Institut d'études slaves, 1997, p. 45-58.

10. Toutes les données concernant l'histoire du théâtre de marionnettes en Tchécoslovaquie dans cet article, indépendantes des prises de position et réactions de Bogatyrëv, sont empruntées au livre de Alice Dubská, Czech Puppets Over the Centuries : An outline of the history of Czech puppeteering up to 1945, Prague : The International Institute of Puppet Arts in Prague, 1998.

11. Bogatyrëv, Češskij kukol'nyj i russkij narodnyj teatr, p. 17.

12. Voir note 2.

13. Le répertoire traditionnel des théâtres de marionnettes dans les pays tchèques, extrêmement composite, comprenait des pièces dérivées de sources aussi différentes que le théâtre élisabéthain, des pièces bibliques et mythologiques, des tragédies germaniques retravaillées pour correspondre au format centre-européen, des opéras italiens et des pièces de la commedia dell'arte.

14. Dubská, Czech Puppets Over the Centuries, et plus particulièrement, p. 21-25.

15. Dubská, Czech Puppets Over the Centuries, et plus particulièrement le chapitre "The amateur puppeteers », p. 25-33.

16. Comme Kasper en Allemagne, Guignol en France ou Petruška en Russie, Kašpárek est le personnage principal du théâtre de marionnettes traditionnel et populaire en pays tchèque. Il incarne un type de héros bon vivant, sans peur et sans reproche. Vif, courageux et généreux, il ne se lasse pas de combattre pour la liberté et la justice.

17. Dubská, Czech Puppets Over the Centuries, et plus particulièrement le chapitre "The amateur puppeteers », p. 33.

18. Bogatyrëv, Češskij kukol'nyj i russkij narodnyj teatr, p. 16.

19. Le Sokol [Faucon] est un mouvement sportif nationaliste tchèque fondé en 1862 qui développa également d'importantes activités culturelles.

20. Bogatyrëv, Češskij kukol'nyj i russkij narodnyj teatr, p. 12.

21. Très proches dans leur aspect grotesque (de grandes oreilles et des yeux globuleux), Spejbl et Hurvínek représentent, pour le premier une figure de " père " à demi éduqué mais ambitieux, pour le second une figure de « fils » inquisiteur et provocateur marqué par la rue.

22. Dubská, Czech Puppets Over the Centuries, et plus particulièrement les chapitres «The development of permanent puppet venues in the 1920s » et « From Kašpárek to Spejbl and Hurvínek », p. 33-39.

23. Voir notes 1 et 2.

24. Dubská, Czech Puppets Over the Centuries, et plus particulièrement le chapitre "The arrival of new development trends in the 1930s », p. 40-44.

25. Bogatyrëv, Češskij kukol'nyj i russkij narodnyj teatr, p. 14-15.

26. Voir note 5 .

27. Bogatyrëv, Češskij kukol'nyj i russkij narodnyj teatr, p. 11.

28. L'Anglais Edward Gordon Craig (1872-1966) fut d'abord metteur en scène puis dut se contenter, par suite de problèmes de financement, de diffuser maquettes de décors et 
costumes. C'est à ce titre qu'il collabora également avec Stanislavskij à une mise en scène de Hamlet à Moscou en 1912, que Bogatyrëv est susceptible d'avoir vue. Craig fit paraître en 1911 son livre De l'art du théâtre qui eut un grand retentissement. Dès 1905, il envisageait la création de "surmarionnettes » censées remplacer les acteurs et il ouvrit en 1913 à Florence une école d'art théâtral qui consacrait une large part au travail avec les marionnettes, mais ferma rapidement lorsque la guerre éclata. Grand collectionneur de marionnettes, Craig publia en 1918-1919 une revue consacrée aux marionnettes, The Marionnette. Voir Christopher Innes, Edward Gordon Craig : A Vision of Theatre, Amsterdam, 1998 ; et Patrick Le Bœuf, éd., Craig et la marionnette, Arles - Paris, 2009. Concernant Craig et Stanislavskij, voir le catalogue d'exposition: Brian Arnott, Towards a New Theatre: Edward Gordon Craig and Hamlet, Ottawa, 1975.

29. Bogatyrëv, Češskij kukol’nyj i russkij narodnyj teatr, p. 13.

30. Bogatyrëv, « Puppentheater in Münster », p. 4.

31. Bogatyrëv, Češskij kukol’nyj i russkij narodnyj teatr, p. 11.

32. Ibid., p. 19-20.

33. Ibid., p. 20.

34. Ibid., p. 25.

35. Pëtr Bogatyrëv et Roman Jakobson, « Slavjanskaja filologija v Rossii za gody vojny $i$ revolujucii [La philologie slave en Russie pendant les années de guerre et la révolution]», Slavia, 1, Prague, 1922, p. 171-184; 457-469; 626-634; puis sous forme de brochure à Berlin : Opojaz, 1923.

36. P. Bogatyrëv, Actes magiques, rites et croyances en Russie subcarpathique, Travaux publiés par l'Institut d'études slaves, XI, P. : Honoré Champion, 1929.

37. Voir à ce sujet Céline Trautmann-Waller, « Étude des cultures populaires et transferts culturels : le livre de Pëtr Bogatyrëv sur la Russie subcarpathique (1929) », à paraitre en russe. Bogatyrëv insiste sur l'actualité des superstitions et des pratiques magiques et montre que celles-ci, loin de se limiter au monde rural, concernent également par exemple les artistes. Un phénomène également souligné est la résurgence des pratiques magiques pendant la révolution russe.

38. Voir Carole Maigné, Céline Trautmann-Waller, éds., Formalismes esthétiques et héritage herbartien : Vienne, Prague, Moscou, Hildeseim - Zürich - New York : Olms, 2009.

39. Voir par exemple l'analyse que fait Klaas-Hinrich Ehlers des contacts entre ethnographie fonctionnelle-structurale et Volkskunde allemande, mais aussi de certaines tendances idéologiques de cette dernière (idéalisation de la paysannerie comme collectivité originaire et « saine ", c'est-à-dire non touchée par les maladies modernes de l'individualisme et du matérialisme), tout à fait contraires aux vues de Bogatyrëv : Klaas-Hinrich Ehlers, « Pëtr Bogatyrëv in Münster. Funktional-strukturale Ethnographie und deutsche Volkskunde im Kontakt », Zeitschrift für Volkskunde, 94 (1), 1998, p. 7-24.

40. Bogatyrëv, Češskij kukol'nyj i russkij narodnyj teatr, p. 29-65.

41. Ibid., p. 70-71.

42. Ibid., p. 71-72.

43. Ibid., p. 75.

44. Ibid., p. 77-78.

45. Ibid., p. 84-86. 
46. Bogatyrëv, « Puppentheater in Münster », p. 3-4.

47. Ibid., p. 4.

48. Bogatyrëv, « Vystavka kukol'nogo teatra SSSR v Prage », p. 5-8.

49. Bogatyrëv, Češskij kukol'nyj i russkij narodnyj teatr, p. 78-81.

50. Ibid., p. 20-28.

51. P. Bogatyrëv et R. Jakobson, «Fol'klor kak osobaja forma tvorčestva [Le folklore comme forme particulière de création]", in P. Bogatyrëv, Voprosy teorii narodnogo iskusstva [De la théorie de l'art populaire], M., 1971; P. Bogatyrëv et R. Jakobson, « Die Folklore als besondere Form des Schaffens ", in Roman Jakobson, Selected Writings IV (Slavic Epic Studies), The Hague : Mouton, 1966, p. 1-15.

52. Petr Kaiser, «Protostrukturalismus in der tschechischen Ästhetik und Literaturwissenschaft : Otakar Zischs Dichtungstypologie », in Wolfgang F. Schwarz, éd., Praguer Schule: Kontinuität und Wandel: Arbeiten zur Literaturästhetik und Poetik der Narration, Francfort am Main, 1997, p. 101-109.

53. Voir Veronika Ambros, « Prague's Experimental Stage : Laboratory of Theatre and Semiotics », Semiotica, 168, 2008, p. 45-65.

54. P. Bogatyrëv, «Znaky divadelní » (traduit en anglais sous le titre : Semiotics in the Folk Theatre), in Matejka, Titunik, éds., Semiotics of Art. Prague School Contributions, p. 3350 ; et du même, "Lidové divadlo české a slovenské » (traduit en anglais sous le titre : Forms and Functions of Folk Theatre), ibid., p. 51-56.

55. P. Bogatyrëv, "Čaplin i “Kid”", in Viktor Šklovskij, éd., Čaplin: Sbornik stat'ej [Chaplin: Recueil d'articles], Berlin : Izdatel'stvo žurnala « Kino », 1923, p. 57-80 ; et du même, « Čaplin - mnimyj graf [Le comte imaginaire] », ibid., p. 81-94.

56. Voir Jarka M. Burian, Leading Creators of Twentieth-Century Czech Theatre, Londres New York : Routledge, 2002, et plus particulièrement le chapitre 3 consacré à E.F. Burian. Voir également les articles sur le théâtre populaire que Bogatyrëv publia dans la revueprogramme fondée par Burian: "Puškin a lidové divadlo " [Puškin et le théâtre populaire], Program D 37, Prague, 1936-1937, p.150-152 ; "Lidové divadlo » [Le théâtre populaire], ibid., p. 188-195; "Lidové divadlo » [Le théâtre populaire], Program D 39, Prague, 1938-39, p. 151-157.

\section{RÉSUMÉS}

Résumé

Pendant son séjour à Prague, et plus précisément entre les années 1923 et 1931, Bogatyrëv a consacré au théâtre de marionnettes un livre (Češskij kukol'nyj i russkij narodnyj teatr [Le théâtre de marionnettes tchèque et le théâtre populaire russe], 1923) et plusieurs articles. L'article commence par étudier ce que les travaux de Bogatyrëv nous révèlent de sa fréquentation des théâtres de marionnettes tchèques et de ses réactions à l'effervescence étonnante qu'ils suscitent à l'époque, liée notamment aux débuts de la jeune République tchécoslovaque, avant de passer aux grandes lignes de son analyse. L'approche des héritiers de l'esthétique néo- 
herbartienne tchèque, attentive aux caractéristiques concrètes du matériau artistique, interagit ici avec celles des traditions ethnographiques russes et du formalisme, tel que Bogatyrëv le connaissait à travers le Cercle linguistique de Moscou et à travers ses contacts avec certains des membres de l'OPOJAZ, mais aussi avec l'inspiration reçue de la Volkskunde allemande de l'époque, en même temps que par la critique lucide de certains de ses aspects, et les travaux de la linguistique et de l'ethnologie française (Meillet, Lévy-Bruhl, Dumézil).

De manière générale le déplacement de l'analyse sur la représentation elle-même en tant que performance ponctuelle, lieu d'échange et entité sémiotique complexe, participe d'une tendance plus large à défendre l'« actualité » des pratiques culturelles contre la "survivance ». Tendue entre deux traditions populaires (le théâtre populaire russe et le théâtre de marionnettes tchèque) et entre les avant-gardes de ces deux mêmes pays, l'étude de Bogatyrëv inclut une réflexion sur les relations entre tradition, improvisation et création, les échanges entre art des élites et art populaire, l'interaction entre le créateur et son public et ouvre sur une analyse de la spécificité du signe théâtral.

\section{Abstract}

Between the years 1923 and 1931, at the time of his stay in Prague, Pëtr Bogatyrëv devoted several articles to puppet theater and an essay, Tcheshskii kukol'nyi i russkii narodnyi teatr (Czech puppet theater and Russian folk theater, 1923). The present article starts with a study of what Bogatyrëv's works reveal about his attendance at Czech puppet theaters and how he reacted to the stunning excitement they sparked at the time - a time of effervescence following the birth of the young Czech Republic. The article then moves on to the analysis per se. The approach adopted by the heirs of the Czech neo-Herbatian esthetics, which focuses on the concrete characteristics of the artistic material, interacts with the approaches of Russian ethnographical traditions and of Formalism as Bogatyrëv knew it through the Moscow Linguistic Circle and his contacts with certain members of OPOIAZ; it also interacts with the German Volkskunde of the time and the lucid criticism of some of its aspects; and last, with the works of French linguists and ethnologists (Meillet, Lévy-Bruhl, Dumézil).

On the whole, the analytic shift toward the performance itself as live performance, a place for exchange and a complex semiotic entity, partakes of a larger tendency to support the "presentday" character of cultural practices against "survivals." Situated midway between two popular traditions (Russian folk theater and Czech puppet theater) and between these two countries' avant-gardes, Bogatyrëv's study includes a reflection on the relations between tradition, improvisation and creation, exchanges between elite and folk art, the interaction between the creator and his public, and marks the beginning of the study of the specificity of the theatrical sign.

\section{AUTEUR}

\section{CÉLINE TRAUTMANN-WALLER}

Université Paris 3 - Sorbonne Nouvelle / IUF, UMR 8547 Pays germaniques, équipe « Transferts culturels » 